\title{
Web based fuzzy diagnosis system and evaluation for five diseases with sort throat symptom
}

\author{
Sadaf Anbarzadeh ${ }^{1}$, Hossein Davari² \\ 1 instructor, Department of Electrical engineering, Sheikh Bahaei University, Baharestan, Isfahan, \\ Iran \\ ${ }^{2}$ B.S student of Electrical engineering, Sheikh Bahaei University, Baharestan, Isfahan, Iran
}

\begin{abstract}
Automatic disease diagnosis has been human concern for a long time. Since people are so busy and the doctors visit expenses are so expensive, a lot of different attempts have been done in the field of design of expert system for disease diagnosis. This paper describes a project work aiming to develop a web based fuzzy expert system for human disease diagnosis. This program models the thinking pattern and human activity and leads to close the expert system and human action method. In this paper we have consulted with different physicians and analyzed the diagnosis procedure and modeled them with a fuzzy expert system. This project is based on development of a web-based clinical tool designed to improve the quality of the exchange of health information between health care professionals and patients. This system has been tested on five diseases with sort throat symptom such as mononucleosis, scarlet fever, pharyngitis or tonsillitis, common cold and virus infection and exhibited satisfactory results.
\end{abstract}

Keywords: expert system, fuzzy expert system, online diagnosis system, knowledge base fuzzy expert system. 


\section{Introduction}

$\mathrm{N}$ owadays one of the problems of human is disease diagnosis by himself and doing self curing that may worsen the disease process and cause problems by drug side effect and also other kind of diseases. This problem incites researcher to design a system that help patient to initially diagnose his disease independently. If patient doesn't access to the physician in a period of time, he can start treatment by himself and after that he can refer to specialist to continue complete and perfect treatment. One of these systems is online disease diagnosis that has been welcomed by more people day by day Because In today's world people are so busy that they hardly have enough time to visit a physician and also they must pay a high price. By this expert diagnosis system, they can have an idea about their diseases in any places such as home or office by themselves and after that refer to the physician and continue their treatment. Moreover this system can help physicians for more accurate and faster diagnosis.

In this paper the designed system was evaluated in five diseases with sort throat symptom. The sort throat is one of the diseases that everybody experienced at least one time a year. Patient starts to medicate most of the times and sometimes he/she may make a mistake. So we survey five widespread diseases with sort throat symptom such as mononucleosis, scarlet fever, pharyngitis or tonsillitis, common cold and virus infection. The result compared with classical diagnosis by specialist.

Different disease diagnosis is one of the most useful researches that have been done recently. This paper has proposed an expert fuzzy system for diagnosis. This method is based on if-then fuzzy rules, receives knowledge and does classifying. Fuzzy logic has the advantages that the solution to the problem can be cast in terms that human operators can understand.

The knowledge that one physician notices can be considered in a fuzzy rule set and improves with optimization algorithm in order to better the confidence level and accuracy of the diagnosis. Furthermore after different clinical and pathological tests, sometimes its too difficult for the physicians to diagnose the disease exactly and can be helped with this system.
This research presents a novel method for online diagnosis. In this method, based on the selection of the problem area/ problem, the expert system gives some symptom from which the user needs to select symptoms. Based on the selection of symptoms, the user is again asked some questions. According to the answer selection, the fuzzy expert system diagnosis diseases based on its knowledge, add catalyst factor (if any), do ranking and gives the result in fuzzy form. As fuzzy expert system deals with uncertainty and vague terms, it is generously accepted in different sphere of life.

\section{Fuzzy system and prior works}

The term "fuzzy logic" was introduced with the 1965 proposal of fuzzy set theory by Lotfi A. Zadeh. Fuzzy logic has been applied to many fields, from control theory to artificial intelligence. Fuzzy logic had, however, been studied since the 1920s, as infinite-valued logicnotably by Łukasiewicz and Tarski.

Fuzzy logic is a form of many-valued logic in which the truth values of variables may be any real number between 0 and 1. By contrast, in Boolean logic, the truth values of variables may only be 0 or 1 . Fuzzy logic has been extended to handle the concept of partial truth, where the truth value may range between completely true and completely false. Furthermore, when linguistic variables are used, these degrees may be managed by specific functions.

Knowledge is a theoretical or practical understanding of a subject or a domain. In other words, Knowledge is the sum of what is currently known. Diagnosis system is a system which can diagnose diseases through checking out the symptoms. A knowledge based online diagnosis system is developed for diagnosis of diseases based on the knowledge given by physicians in the system.

A computer Program Capable of performing at a human-expert level in a narrow problem domain area is called an expert system. Management of uncertainty is an intrinsically important issue in the design of expert systems because much of the information in the 
knowledge base of a typical expert system is imprecise, incomplete or not totally reliable. An expert system that uses fuzzy logic instead of Boolean logic is known as Fuzzy expert system.

A fuzzy expert system is a collection of fuzzy rules and membership functions that are used to reason about data. Using fuzzy expert system expert knowledge an be represented that use vague and ambiguous terms in computer.

Fuzzy logic is a set of mathematical principles for knowledge representation based on degrees of membership rather than the crisp membership of classical binary logic. Unlike two-valued Boolean logic, fuzzy logic is multi valued. Fuzzy logic is a logic that describes fuzziness. As fuzzy logic attempts to model human's sense of words, decision making and common sense, it is leading to more human intelligent machines.

The National Research Council, Canada has developed an online diagnosis system. Initially, they used acute leukaemia disease data to illustrate the efficacy of the diagnostic tool. More recently, they developed a new fuzzy classification method called PROAFTN to assist medical diagnosis. Results obtained using the PROAFTN method on acute leukaemia diagnosis are promising. The challenge herein is to provide secure, remote access, to decision support tools and a standard framework for the exchange of health information, over inexpensive Internet communication pathways using web-based technologies.

Informantion related to this can be found from the website of National Research Council Canada.

YourDiagnosis is developed by an expert panel of very experienced doctors in Australia. They are part of a large medical and hospital group called Macquarie Health Corporation Ltd. Macquarie Health Corporation Ltd. also owns Macquarie Hospital Services and Macquarie Medical Systems. Macquarie Hospital Services has owned and operated private hospitals since 1976.

Easy Diagnosis is a online diagnosis system. This expert system software provides a list and clinical escription of the most likely conditions based on an analysis of patient's particular symptoms. EasyDiagnosis focuses on the most common medical complaints that account for the majority of physician visits and hospitalizations.

Wrong Diagnosis which is part of Health Grades network and WebMD whose slogan is "Better Information, Better Health" are another two efficient online diagnosis tools.

There are many online diagnosis tool in the web, but proposed system is the most user friendly (easy to use), precise, at the same time accurate. In the web, some systems are so boring -needs to give lots of information, give answers to lots of question which is quite monotonous. In most of the other online diagnosis websites, the sequence of questions is fixed.

But in the proposed system one needs to give minimal information, questions will be asked after filtering at every step based on your responses and at every step the problem domain will become narrower to reach a perfect diagnosis.

\section{Short description about five selected diseases}

We have selected five diseases to evaluate our system. In this part we have short description about these diseases:

Infectious mononucleosis (IM; also known as mono, glandular fever, Pfeiffer's disease, Filatov's disease, and sometimes colloquially as the kissing disease from its transmission by saliva) is an infectious, widespread viral disease most commonly caused by the Epstein-Barr virus (EBV), one type of herpes virus, against which over $90 \%$ of adults are likely to have acquired immunity by the age of 40. Occasionally, the symptoms can reoccur at a later period. Most people are exposed to the virus as children, when the disease produces no noticeable or only flu-like symptoms. Especially in adolescents and young adults, the disease is characterized by fever, sore throat and fatigue, along with several other possible signs and symptoms.

Scarlet fever (also called scarlatina in older literature) is an infectious disease which most commonly affects children. Signs and symptoms include sore throat, fever, and a characteristic red rash. Scarlet fever is usually spread by inhalation. There is no vaccine, but the disease is 
effectively treated with antibiotics. Most of the clinical features are caused by erythrogenic toxin, a substance produced by the bacterium Streptococcus pyogenes (group A streptococcus) when it is infected by a certain bacteriophage.

Pharyngitis is the inflammation of the pharynx, a region in the back of the throat. In most cases it is quite painful, and it is the most common cause of a sore throat.

Tonsillitis is inflammation of the tonsils most commonly caused by viral or bacterial infection. Symptoms may include sore throat and fever. When caused by a bacterium belonging to the group A streptococcus, it is typically referred to as strep throat. The overwhelming majority of people recover completely, with or without medication. In $40 \%$, symptoms will resolve in three days, and within one week in $85 \%$ of people, regardless of whether streptococcal infection is present or not.

\section{Proposed disease diagnosis expert fuzzy system}

We have met different specialist and asked them about different diseases that might be behind the sort throat symptom. After consulting five diseases are selected that more probable. They are mononucleosis, scarlet fever, pharyngitis or tonsillitis, common cold and virus infection. Then we asked the physicians about other probable symptoms that might appear, asked them about key factors and questions that determine the exact disease. Finally we have found that all of them have a same procedure and modeled their method.(figure .1) .

Then a lot of information (symptom, signs etc.) of different diseases have been collected for particular category as sample $[8,9,10]$ and Made a comparative analysis to identify which symptoms are major symptoms for particular diseases i.e. that symptom must be present, their acuity level etc.

Then a uniform structure is made and a mathematical equivalence (equation (1-3)) is formed, which will be used to diagnosis by the fuzzy expert system.

After that implementation of the expert system is done and tested it thoroughly to check whether the desired result is coming or not.
After necessary corrections, when it seems ok with respect to the desired goal, then consulted with the physicians again and explained about the software implementation and showed it to them and they acknowledged it, gave some suggestions(to add catalyst factor which is some sort of personal information/ patient's history information), according to which the system is revised.

sweighted $=\sum$ symi $* W i$

smajor $=\sum($ Smajor $) j($ Wmax $\left.) j\right)$

disease probability $=\frac{(\text { sweighted }- \text { smajor }- \text { low })}{\text { high }- \text { low }} * 100 \%+\sum$ cat

counters $=\mathrm{i}, \mathrm{j}$

$\mathrm{Sym}_{\mathrm{i}}=$ the symptom selected or not

$\mathrm{W}_{\mathrm{i}}=$ weight of symptom

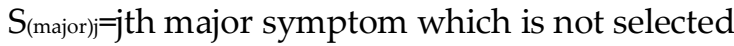

high $=$ Maximum range of disease

low $=$ Minimum range of disease

Sweighted=total selected

smajor=Total not selected

Cat $=$ catalyst factor

The proposed expert system assist user to diagnosis diseases, he/she might have, in a fuzzy way. Based on the selection of the problem area/problem, the expert system gives some symptom from which the user needs to select symptoms, based on the selection of symptoms the user is asked some questions, according to the answer selection the fuzzy expert system diagnosis diseases based on it's knowledge, add catalyst factor (if any) and do ranking and gives result in fuzzy form. 

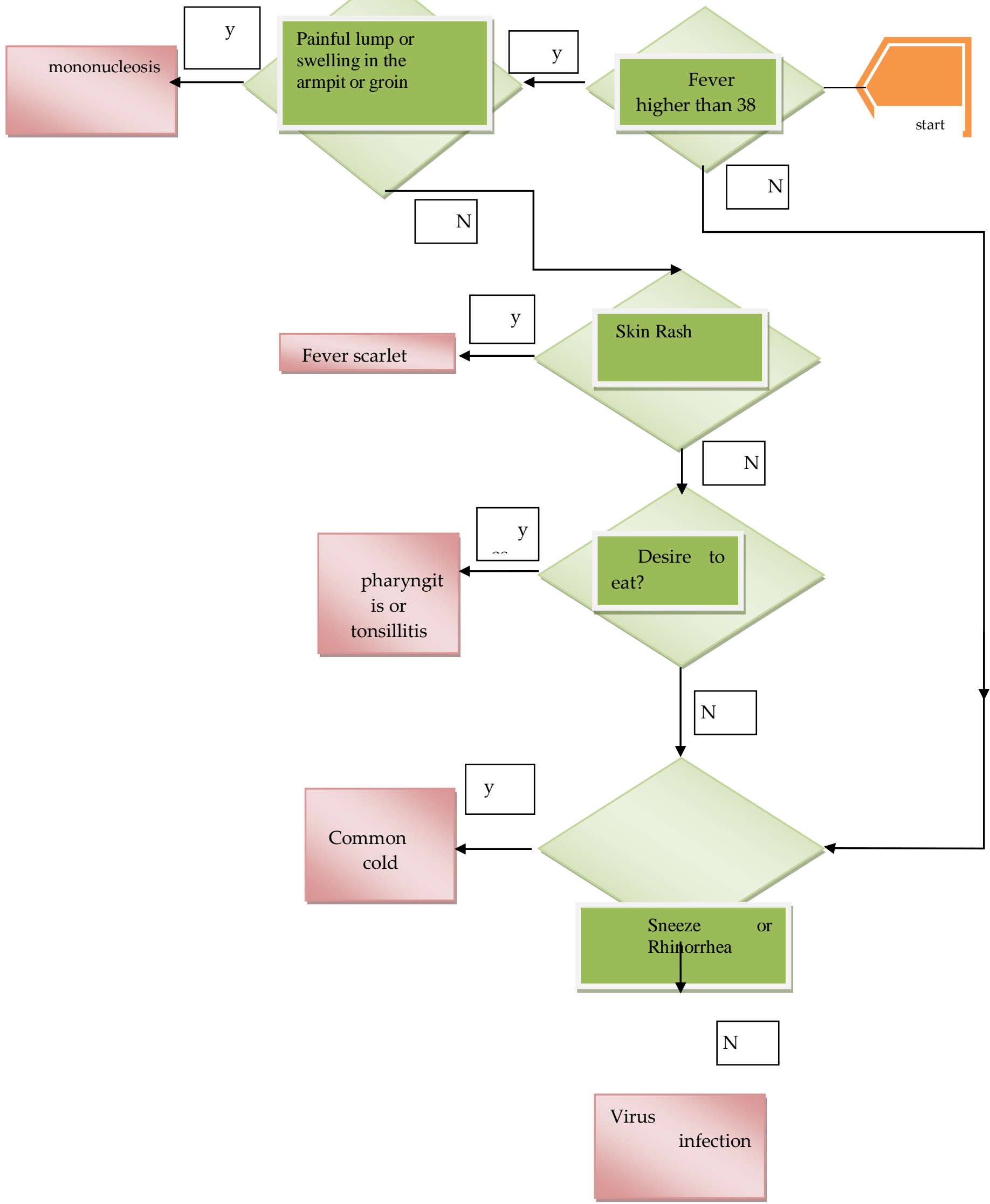

Figure1: Modeling of diagnosis process for 5 diseases with sort throat symptom 


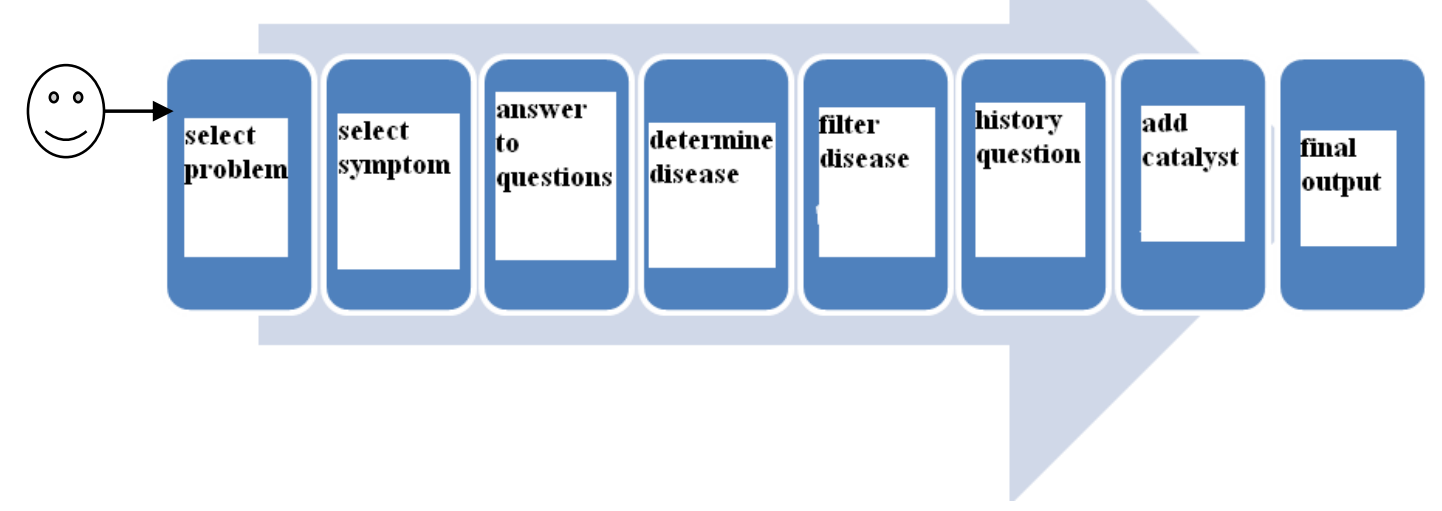

Figure2: Flow diagram

In the proposed system sort throat disease is tested. If the user select sort throat five questions will be asked that are related symptoms corresponding to the interview and consultation with the expert physicians:

- Fever

- Skin Rash

- Desire to eat

- Sneeze or Rhinorrhea

- Painful lump or swelling in the armpit or groin

According to the symptoms, five diseases are diagnosed. These are as mononucleosis, scarlet fever, pharyngitis or tonsillitis, common cold and virus infection.

\section{Evaluation of the proposed system}

Fuzzy logic is a logical calculus which operates with many truth values (while classical logic works with the two values of true and false). Since fuzzy logic considers the truth of scientific statements like something softened, it is fruitfully applied to the study of biological phenomena; biology is indeed considered the field of complexity, uncertainty and vagueness. In this paper fuzzy logic is successfully applied to the clinical diagnosis of a patient who suffers from sort throat diseases. In this work it is presented a mathematical foundation of fuzzy

logic (with connectives and inference rules) and then the application of fuzzy reasoning to the study of a clinical case. Probabilistic logic is widely considered the unique logical calculus useful in clinical diagnosis, thus the usefulness of fuzzy logic and its relation with probabilistic logic is here explored.
A computer Program Capable of performing at a human expert level in a narrow problem domain area is called an expert system. Basic characteristics of expert systems are:

Use knowledge rather than data, Knowledge is encoded \& maintained as an entity, separate from the control program, Capable of explaining how a particular conclusion is reached and why requested information is needed, Use symbolic representation and perform inference through symbolic computation

Now if the characteristics of the proposed system are observed, it is seen that, it performs in a narrow problem domain area (only diagnosis diseases) at a human expert level (like physicians). It uses knowledge to make diagnosis decision; the knowledge is given by some expert physicians. It can also explain how a particular decision made, that is why the proposed system is an expert system. As it is using knowledge base in making decision, so it is also knowledge based expert system.

Let the user select sort throat as his/her problem area. Assume there are 5 diseases under category mononucleosis, scarlet fever, pharyngitis or tonsillitis, common cold and virus infection. Then all the symptoms under that will be shown to the user by the expert system which are given in row one of Table 1 . Then the user will select symptoms applicable to him/ her. After that the user will need to answer some question given by the expert system on selected symptoms. Based on the given answer and answer of history question in this case, the user need to answer two history question, in this particular case the catalyst factor is 4 and they belongs to scarlet fever) which is termed as catalyst factor, the expert system computes the probabilities of probable diseases and filter them before showing. The total diagnosis scenario is depicted in Table 1. 
Table 1.symptom units

\begin{tabular}{|c|c|c|c|c|c|}
\hline diseases / symp & $\mathbf{1}$ & $\mathbf{2}$ & $\mathbf{3}$ & $\mathbf{4}$ & $\mathbf{5}$ \\
\hline mononucleosis & 0.9 & 0 & 0.5 & 0 & 0.9 \\
\hline scarlet fever & 0.8 & 0.9 & 0.4 & 0 & 0 \\
\hline $\begin{array}{c}\text { pharyngitis or } \\
\text { tonsillitis }\end{array}$ & 0.9 & 0 & 0.9 & 0 & 0 \\
\hline common cold & 0.7 & 0 & 0.3 & 0.9 & 0 \\
\hline virus infection & 0.5 & 0 & 0.2 & 0.3 & 0 \\
\hline
\end{tabular}

The first row is the diseases symptoms:

1.Fever

2.Skin Rash

3.Desire to eat

4.Sneeze or Rhinorrhea

5.Painful lump or swelling in the armpit or groin

For example:

Sweighted=2.3, $\Sigma($ Smajor $) \mathrm{j}($ Wmajor $) \mathrm{j}=0$, high $=$ 3.3 low $=0.2$, Cat $_{\mathrm{f}}=4$

$$
=4+\frac{2.3-0-0.2}{3.3-0.2}=\% 71
$$

mononucleosis probability

sweighted=2.1, $\quad \Sigma($ Smajor $) \mathrm{j}($ Wmajor $) \mathrm{j}=0$, high $=4.5$, low $=0.8$, Cat $_{\mathrm{f}}=0$

scarlet fever probability $=\frac{2.1-0-0.8}{4.5-0.8}=35 \%$

sweighted $=1.8, \Sigma($ Smajor $) \mathrm{j}($ Wmajor $) \mathrm{j}=0$, high=3.6, low $=0.4$, Catf $=0$

pharyngitis or tonsillitis

probability $=\frac{1.8-0-0.4}{3.6-0.4}=43 \%$

sweighted $=1.9, \Sigma($ Smajor $) j($ Wmajor $) j=0$, high=2, low $=0.7$, Catf $=$

$$
0 \quad=\frac{1.9-0-0.7}{2-0.7}=92 \%
$$

common cold probability

sweighted $=1 \sum$ (Smajor)j(Wmajor $) \mathrm{j}=0$, high=4.2, low $=0.5$, Catf $=0$

virus infection probability $=\frac{1-0-0.5}{4.2-0.5}=13 \%$

The following chart (Figure. 3) gives a quick overview of the confidence level of the proposed system with real time diagnosis. The bar on right for every disease indicates the confidence level of our system without having observation on any pathological test results. And the bar on left shows the full confidence of diagnosis in real time with observing all necessary pathological tests. In our system the confidence level decreases with the increase of number of pathological tests needed.

\section{Conclusions}

The expert diagnosis system helps to save time and expenses. Moreover the patient can start to be cured with an online expert system easier and faster and also refer to the specialist for complete treatment. As this system is knowledge based, it can help physicians to improve confidence level of diagnosis. Of course this system is experimental and can be improved by expert specialists' feedback.

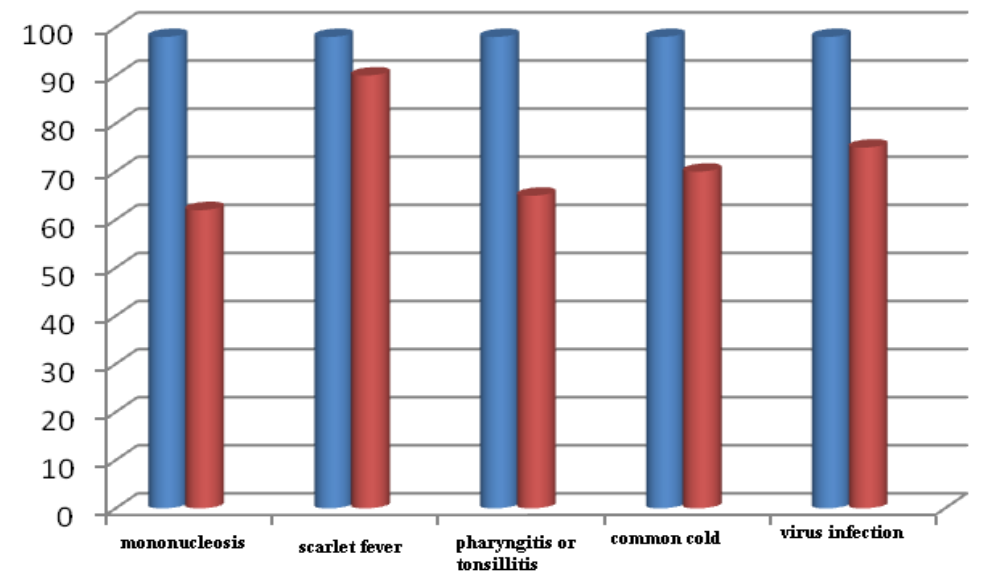

Figure 3: Confidence level 


\section{References}

D. W. Patterson, (1990) "Introduction to Artificial Intelligence and Expert Systems", PrenticeHall Inc., Englewood Cliffs, N.J, USA

Easydiagnosis, an online diagnosis tool," Available: http://easydiagnosis.com/ (Last Accessed Date:15-04-10).

J. Macleod, J. Munro, C. Edwards and C. Edwards, (1990) "Macleod's clinical examination". Churchill Livingstone.

M. Negnevitsky, (2005) “Artificial intelligence: A guide to intelligent systems", Addison Wesley Longman.

National research council canada," Available: http://www.nrccnrc.gc.ca/ eng/index.html (Last Accessed Date:15-04-10)

Shi, Y. and Eberhart, R. and Chen, Y., (1999) "Implementation of evolutionary fuzzy systems", IEEE Transactions on Fuzzy Systems, Vol. 7, No. 2, pp 109-119

Symptoms of different diseases," Available: http://www.wrong diagnosis.com/ (Last Accessed Date:15-04-10)

Symptoms of different diseases," Available: http://www.webmd.com/ (Last Accessed Date:15-04-10)

Yourdiagnosis, an online diagnosis tool," Available: http://www.yourdiagnosis.com/ (Last Accessed Date:15-04-10).

Zadeh,LA, (1983) "The role of fuzzy logic in the management of uncertainty in expert systems", Fuzzy sets and Systems On Elsevier ,Vol. 11, No. 1-3, pp 197-198 